\title{
CONVERGENCE ESTIMATES AND APPROXIMATION SOLVABILITY OF NONLINEAR IMPLICIT VARIATIONAL INEQUALITIES
}

\author{
RAM U. VERMA \\ International Publications USA \\ 10246 Coed Drive, Suite A-29 \\ Orlando, Florida 32826 USA
}

(Received March, 2000; Revised March, 2001)

\begin{abstract}
Approximation-solvability of a class of nonlinear implicit variational inequalities involving a class of partially relaxed monotone mappings - a computation-oriented class in a Hilbert space setting- is presented with some applications.

Key words: Partially Strongly Monotone Mapping, Partially Relaxed Monotone Mapping, Approximation-Solvability, Partially Monotone Mapping.
\end{abstract}

AMS subject classifications: $49 \mathrm{~J} 35$.

\section{Introduction}

Recent prolific growth in applications of variational inequalities to problems arising from applied mathematics, mathematical programming, optimization and control theory, engineering sciences, and others, by any measure, has been outstanding. Verma [14], motivated by the ongoing research on the approximation-solvability of variational inequalities, specially by the works of Cohen [2, 3], Marcotte and $\mathrm{Wu}$ [10] and $\mathrm{Zu}$ and Marcotte [18], introduced a class of partially relaxed monotone mappings and applied them to the approximation-solvability of nonlinear variational inequalities using a general class of iterative algorithms expressed as variational inequalities in a Hilbert or a Banach space setting. The notion of the partial relaxed monotonicity is weaker than the existing notion of cocoercivity [4 10] studied by Marcotte and $\mathrm{Wu}$ [10], $\mathrm{Zu}$ and Marcotte [18], and others in context of the approximation-solvability of a class of variational inequalities in $R^{n}$. The notion of the cocoercivity is also referred to the Dunn property [4].

This paper is concerned with the approximation-solvability based on an iterative algorithm - a modified version of the algorithm [10] characterized as an implicit variational inequality of a class of nonlinear implicit variational inequalities involving a class of partially relaxed monotone mappings in a Hilbert space. An application to $R^{n}$ space is also discussed. To learn more details about the general variational inequalities and related algorithmic applications, see [1-18]).

Let $H$ be a real Hilbert space with the inner product $\langle\cdot, \cdot\rangle$ and norm $\|\cdot\|$. Let $T: K \times K \rightarrow H$ be any mapping and $K$ a closed convex subset of $H$. We consider a class of nonlinear implicit variational inequality (abbreviated as NIVI) problems: determine an element $x^{*} \in K$ such that

$$
\left\langle T\left(x^{*}, x^{*}\right), x-x^{*}\right\rangle \geq 0 \text { for all } x \in K,
$$


which is equivalent to a projection formula

$$
x^{*}=P_{K}\left[x^{*}-\rho T\left(x^{*}, x^{*}\right)\right],
$$

where $P_{K}$ is the projection of $H$ onto $K$, and $\rho>0$ is a constant.

Now we need to recall the following auxiliary results, which are crucial to the development of the work on hand.

Lemma 1.1: An element $u \in K$ is a solution of the NIVI problem (1.1) if and only if

$$
u=P_{K}[u-\rho T(u, u)] \text { for } \rho>0 \text {, }
$$

where $T: K \times K \rightarrow H$ is a mapping and $P_{K}$ is the projection of $H$ onto $K$.

Lemma 1.2: An element $u \in K$ is a solution of the NIVI problem (1.1) if

$$
\langle T(u, u), x-u\rangle \geq 0 \text { for all } x \in K .
$$

A mapping $T: H \rightarrow H$ is said to be $\alpha$-cocoercive [15] if for all $x, y \in H$, we have

$$
\|x-y\|^{2} \geq \alpha^{2}\|T(x)-T(y)\|^{2}+\|\alpha(T(x)-T(y))-(x-y)\|^{2},
$$

where $\alpha>0$ is a constant.

Alternatively, a mapping $T: H \rightarrow H$ is called $\alpha$-cocoercive $[4,10]$ if there exists a constant $\alpha>0$ such that

$$
\langle T(x)-T(y), x-y\rangle \geq \alpha\|T(x)-T(y)\|^{2} \text { for all } x, y \in H .
$$

$T$ is called $r$-strongly monotone if for each $x, y \in H$, we have

$$
\langle T(x)-(y), x-y\rangle \geq r\|x-y\|^{2} \text { for a constant } r>0 .
$$

This implies that

$$
\|T(x)-T(y)\| \geq r\|x-y\|
$$

that is, $T$ is $r$-expanding, and when $r=1$, it is expanding. The mapping $T$ is called monotone if

$$
\langle T(x)-T(y), x-y\rangle \geq 0 \text { for all } x, y \in H .
$$

A mapping $T$ is called $\beta$-Lipschitz continuous (or $\beta$-Lipschitzian) if there exists a constant $\beta \geq 0$ such that

$$
\|T(x)-T(y)\| \leq \beta\|x-y\| \text { for all } x, y \in H .
$$

Lemma 1.3: [10] For any two elements $u, v \in H$, we have

$$
\|u\|^{2}+\langle u, v\rangle \geq(-1 / 4)\|v\|^{2} .
$$

Next, we recall the notions of $r$-partially strongly monotone and $\gamma-r$-partially relaxed monotone mappings that seem to be computation-oriented and are tailored to approximationsolvability of nonlinear variational inequalities and related fields.

A mapping $T: H \rightarrow H$ is said to be $r$-partially strongly monotone if there exists a constant $r>0$ such that 


$$
\langle T(x)-T(y), z-y\rangle \geq r\|x-y\|^{2} \text { for all } x, y, z \in H
$$

For $r=0, T$ is said to be 0-partially monotone, and when $z=x, T$ is $r$-strongly monotone.

A mapping $T: H \rightarrow H$ is called $\gamma-r$-partially relaxed monotone if there exist constants $\gamma, r>0$ such that

$$
\langle T(x)-T(y), z-y\rangle \geq-\gamma\|z-x\|^{2}+r\|x-y\|^{2} \text { for all } x, y, z \in H
$$

The mapping $T$ is referred to $\gamma$-partially relaxed monotone if there exists a constant $\gamma>0$ such that

$$
\langle T(x)-T(y), z-y\rangle \geq-\gamma\|z-x\|^{2} \text { for all } x, y, z \in H
$$

Example 1.1: [14] Consider an $\alpha$-cocoercive mapping $T: H \rightarrow H$. For each $x, y, z \in H$, we have

$$
\begin{gathered}
\langle T(x)-T(y), z-y\rangle=\langle T(x)-T(y), x-y\rangle+\langle T(x)-T(y), z-x\rangle \\
\geq \alpha\|T(x)-T(y)\|^{2}+\langle T(x)-T(y), z-x\rangle \\
=\alpha\left\{\|T(x)-T(y)\|^{2}+(1 / \alpha)\langle T(x)-T(y), z-x\rangle\right\} \\
\geq-(1 / 4 \alpha)\|z-x\|^{2} \text { (by Lemma 1.3). }
\end{gathered}
$$

That means, every $\alpha$-cocoercive mapping $T$ is $(1 / 4 \alpha)$-partially relaxed monotone.

For the general class of relaxed monotone mappings introduced by Verma [14-16], we have the following implications

$$
\begin{gathered}
\text { the } r \text {-partial strong montonicity } \\
\text { the } \gamma-r \text {-partial relaxed monotonicity } \\
\qquad \begin{array}{l}
\downarrow \\
\text { the } \gamma \text {-partial relaxed monotonicity. }
\end{array}
\end{gathered}
$$

\section{Solvability of NIVI (1.1)}

We now consider the approximation-solvability of the NIVI problem (1.1) based on a modified version of the iterative algorithm [10], which is represented by an implicit variational inequality.

Algorithm 2.1: For an arbitrarily chosen initial point $x^{0} \in K$, we consider an iterative algorithm generated as follows (for $k \geq 0$ ):

$$
\begin{gathered}
\left\langle\rho T\left(x^{0}, x^{0}\right)+x^{1}-x^{0}, x-x^{1}\right\rangle \geq 0 \\
\vdots \\
\left\langle\rho T\left(x^{k}, x^{k}\right)+x^{k+1}-x^{k}, x-x^{k+1}\right\rangle \geq 0 \text { for all } x \in K \text { and for } \rho>0 .
\end{gathered}
$$

Algorithm 2.1 is equivalent to the projection formula 


$$
\left.\left.x^{k+1}=P_{K}\right] x^{k}-\rho T\left(x^{k}, x^{k}\right)\right],
$$

where $P_{K}$ is the projection of $H$ onto $K$.

Before we discuss our main result on the approximation-solvability of the NIVI problem (1.1), we need to recall the following auxiliary result.

Lemma 2.1: For $v, w \in H$, we have

$$
\langle v, w\rangle=(1 / 2)\left[\|v+w\|^{2}-\|v\|^{2}-\|w\|^{2}\right] .
$$

Now, we present (based on Algorithm 2.1), the approximation-solvability of the NIVI problem (1.1) involving a combination of $\gamma$-partially relaxed monotone and monotone mappings in a Hilbert space setting.

Theorem 2.1: Let $H$ be a real Hilbert space and $K$ a nonempty closed convex subset of $H$. Let $T: K \times K \rightarrow H$ be $\gamma$-partially relaxed monotone in its second variable and 0 partially monotone in its first variable. Suppose that $T$ is s-Lipschitz continuous in either variable, $x^{*} \in K$ a solution of the NIVI problem (1.1) and the sequence $\left\{x^{k}\right\}$ is generated by Algorithm 2.1. Then we have:

(a) The estimate

$$
\left\|x^{k+1}-x^{*}\right\|^{2} \leq\left\|x^{k}-x^{*}\right\|^{2}-(1-2 \rho \gamma)\left\|x^{k+1}-x^{k}\right\|^{2} .
$$

(b) The sequence $\left\{x^{k}\right\}$ converges to $x^{*}$ for $0<\rho<1 / 2 \gamma$.

Proof: First, we compute the estimate and then show the convergence of the sequence $\left\{x^{k}\right\}$ to $x^{*}$, a solution of the NIVI problem (1.1). Since $x^{k}$ satisfies Algorithm 2.1, we have

$$
\left\langle\rho T\left(x^{k}, x^{k}\right)+x^{k+1}-x^{k}, x-x^{k+1}\right\rangle \geq 0 \text { for all } x \in K .
$$

On the top of that, $x^{*}$ is a solution of the NIVI problem (1.1), that is, we can have, for a constant $\rho>0$ that

$$
\left\langle\rho T\left(x^{*}, x^{*}\right), x-x^{*}\right\rangle \geq 0 \text { for all } x \in K .
$$

Replacing $x$ by $x^{*}$ in (2.1) and $x$ by $x^{k+1}$ in (2.2), and adding, we obtain

$$
\begin{gathered}
0 \leq-\rho\left\langle T\left(x^{k}, x^{k}\right)-T\left(x^{*}, x^{*}\right), x^{k+1}-x^{*}\right\rangle+\left\langle x^{k+1}-x^{k}, x^{*}-x^{k+1}\right\rangle \\
=-\rho\left\langle T\left(x^{k}, x^{k}\right)-T\left(x^{k}, x^{*}\right), x^{k+1}-x^{*}\right\rangle-\left\langle T\left(x^{k}, x^{*}\right)-T\left(x^{*}, x^{*}\right), x^{k+1}-x^{*}\right\rangle \\
+\left\langle x^{k+1}-x^{k}, x^{*}-x^{k+1}\right\rangle
\end{gathered}
$$

Since $T$ is $\gamma$-partially relaxed monotone in the second variable and 0-partially monotone in the first variable, it implies that

$$
0 \leq\left\langle x^{k+1}-x^{k}, x^{*}-x^{k+1}\right\rangle+\rho \gamma\left\|x^{k-1}-x^{k}\right\|^{2} .
$$

Taking $v=x^{k+1}-x^{k}$ and $w=x^{*}-x^{k+1}$ in Lemma 2.1, and applying to (2.3) yields

$$
0 \leq(1 / 2)\left[\left\|x^{*}-x^{k}\right\|^{2}-\left\|x^{k+1}-x^{k}\right\|^{2}-\left\|x^{*}-x^{k+1}\right\|^{2}\right]+\rho \gamma\left\|x^{k-1}-x^{k}\right\|^{2} .
$$

It follows that

$$
\left\|x^{k+1}-x^{*}\right\|^{2} \leq\left\|x^{k}-x^{*}\right\|^{2}-\left\|x^{k+1}-x^{k}\right\|^{2}+2 \rho \gamma\left\|x^{k+1}-x^{k}\right\|^{2} .
$$


That means, we have

$$
\left\|x^{k+1}-x^{*}\right\|^{2} \leq\left\|x^{k}-x^{*}\right\|^{2}-(1-2 \rho \gamma)\left\|x^{k+1}-x^{k}\right\|^{2} .
$$

In light of (2.4), it follows that either

$$
\lim _{k \rightarrow \infty}\left\|x^{k}-x^{*}\right\|=0
$$

or

$$
\lim _{k \rightarrow \infty}\left\|x^{k+1}-x^{k}\right\|=0 .
$$

Under the first alternative, $x^{k} \rightarrow x^{*}$ and $\lim _{k \rightarrow \infty}\left\|x^{k+1}-x^{k}\right\|=0$ as well. If we consider the second one, then assume that $x^{\prime}=\lim _{k \in K} x^{k}$ is the cluster point of a convergent subsequence. Since the left-hand term of (2.4) is bounded, $x^{\prime}$ must exist. Next, the continuity of the projection mapping (in light of the $s$-Lipschitz continuity of $T$ in either variable) defined by

$$
x^{k+1}=P_{K}\left[x^{k}-\rho T\left(x^{k}, x^{k}\right)\right],
$$

ensures that $x^{\prime}$ is a fixed point of the projection mapping and, as a result, $x^{\prime}$ is a solution of the NIVI problem (1.1). Thus the entire sequence converges to $x^{\prime}$. This completes the proof.

\section{An Application}

In this section we consider the convergence of a symmetric projection method, similar to that of Marcotte and $\mathrm{Wu}$ [10]. Let $F: X \times X \rightarrow R^{n}$ be a mapping from $X \times X$ into $R^{n}$, where $X$ is a closed convex subset of $R^{n}$.

We consider an implicit variational inequality problem: find an element $u \in X$ such that

$$
[F(u, u)]^{t}(x-u) \geq 0 \text { for all } x \in X,
$$

where $[F(u, u)]^{t}$ denotes the transpose of the vector $F(u, u)$. Based on Algorithm 2.1, we have:

Algorithm 3.1: For an arbitrary chosen initial point $x^{0} \in X$, a sequence $\left\{x^{k}\right\}$ is generated by an iterative scheme:

$$
\left[\rho F\left(x^{k}, x^{k}\right)+D_{\rho}\left(x^{k+1}-x^{k}\right)\right]^{t}\left(x-x^{k+1}\right) \geq 0 \text { for all } x \in X,
$$

where $D_{\rho}$ is a fixed positive-definite matrix.

In what follows, $D_{\rho}$ shall denote a symmetric matrix in (3.2) for the convergence of the projection method. The symbols $\lambda_{\min }(S)$ and $\lambda_{\max }(S)$ shall denote, respectively, the minimum and maximum eigenvalues of a symmetric matrix $S$.

Since $D_{\rho}$ is symmetric, it implies that (3.2) is equivalent to

$$
x^{k+1}=P_{D_{\rho}}\left[x^{k}-D_{\rho}^{-1}\left(\rho F\left(x^{k}, x^{k}\right)\right)\right],
$$

where $P_{D_{\rho}}$ is the projection on the set $X$ with respect to the norm $\|\cdot\| D_{\rho}$ induced by the positive-definite symmetric matrix $D_{\rho}$. 
Theorem 3.1: Let $F$ be $\gamma$-partially relaxed monotone in the second variable and 0partially monotone in the first variable, and $D_{\rho}=D$, where $D$ is a symmetric positivedefinite matrix. Suppose that $T$ is s-Lipschitz continuous in either variable, sequence $\left\{x^{k}\right\}$ is generated by Algorithm 3.1 for a constant $\rho>0$ and $x^{*}$ is a solution

of the implicit variational inequality (3.1). Then we have the following conclusions:

(i) $\left.\quad\left\|x^{k+1}-x^{*}\right\|_{D}^{2} \leq\left\|x^{k}-x^{*}\right\|_{D}^{2}-\left[1-2 \rho \gamma \lambda_{\max } D\right)\right]\left\|x^{k+1}-x^{k}\right\|_{D}^{2}$.

(ii) The sequence $\left\{x^{k}\right\}$ generated by Algorithm 3.1 converges to $x^{*}$, a solution of the implicit variational inequality (3.1) for $0<\rho<1 / 2 \gamma \lambda_{\max }(D)$.

Proof: The proof is similar to that of Theorem 2.1.

\section{References}

[1] Baiocchi, C. and Capelo, A., Variational and Quasivariational Inequalities, Wiley \& Sons, New York 1984.

[2] Cohen, G., Auxiliary problem principle and decomposition of optimization problems, $J$. Optim. Theo. Appl. 32:3 (1980), 277-305.

[3] Cohen, G., Auxiliary problem principle extended to variational inequalities, J. Optim. Theo. Appl. 59:2 (1988), 325-333.

[4] Dunn, J.C., Convexity, monotonicity and gradient processes in Hilbert spaces, J. Math. Anal. Appl. 53 (1976), 145-158.

[5] Guo, J.S. and Yao, J.C., Extensions of strongly nonlinear quasivariational inequalities, Appl. Math. Letters 53:3 (1992), 35-38.

[6] He, B.S., A new method for a class of linear variational inequalities, Math. Progr. 66 (1994), 137-144.

[7] Huang, N.J., Generalized nonlinear implicit quasivariational inclusion and an application to implicit variational inequalities, ZAMM 79:8 (1999), 569-575.

[8] Kinderlehrer, D. and Stampacchia, G., An Introduction to Variational Inequalities and their Applications, Academic Press, New York 1980.

[9] Korpelevich, G.M., The extragradient method for finding saddle points and other problems, Matecon 12 (1976), 747-756.

[10] Marcotte, P. and Wu, J., On the convergence of projection methods, J. Optim. Theo. Appl. 85 (1995), 347-362.

[11] Pang, J.S. and Chan, D., Iterative methods for variational and complementarity problems, Math. Progr. 24 (1982), 284-313.

[12] Verma, R.U., Nonlinear variational and constrained hemivariational inequalities involving relaxed operators, ZAMM 77:5 (1997), 387-391.

[13] Verma, R.U., RKKM mapping theorems and variational inequalities, Math. Proc. Royal Irish Acad. 98A:2 (1998), 131-138.

[14] Verma, R.U., Approximation-solvability of nonlinear variational inequalities involving partially relaxed monotone (prm) mappings, Adv. Nonl. Variat. Ineq. 2:2 (1999), 137148.

[15] Verma, R.U., Auxiliary problem principle and its extension applied to variational inequalities, Math. Sci. Res. Hot-Line 3:12 (1999), 7-26.

[16] Verma, R.U., General auxiliary problem principle applied to solvability of nonlinear variational inequalities involving a class of partially relaxed monotone mappings, $A d v$. Nonl. Variat. Ineq. 31:1 (2000), 135-154.

[17] Zeidler, E., Nonlinear Functional Analysis and its Applications I, Springer-Verlag, New York 1986.

[18] Zu, D.L. and Marcotte, P., Co-coercivity and its role in the convergence of iterative schemes for solving variational inequalities, SIAM J. Optim. 6:3 (1996), 714-726. 


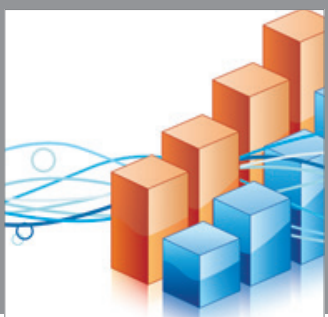

Advances in

Operations Research

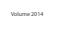

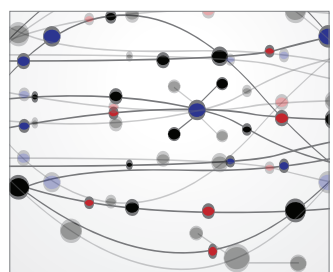

\section{The Scientific} World Journal
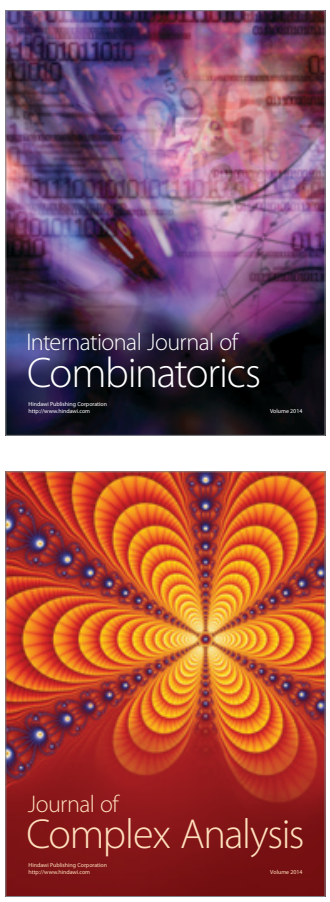

International Journal of

Mathematics and

Mathematical

Sciences
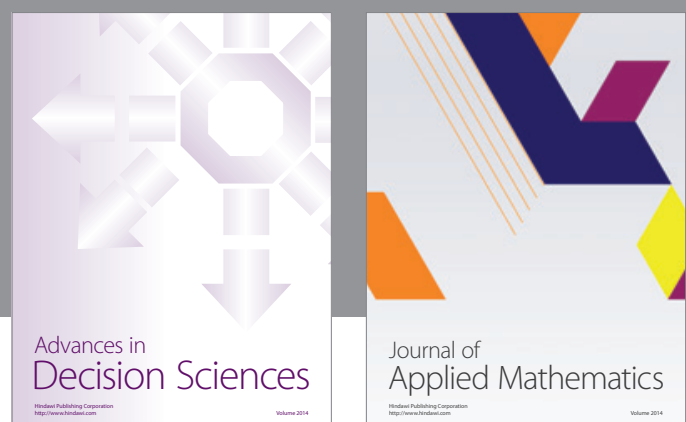

Journal of

Applied Mathematics
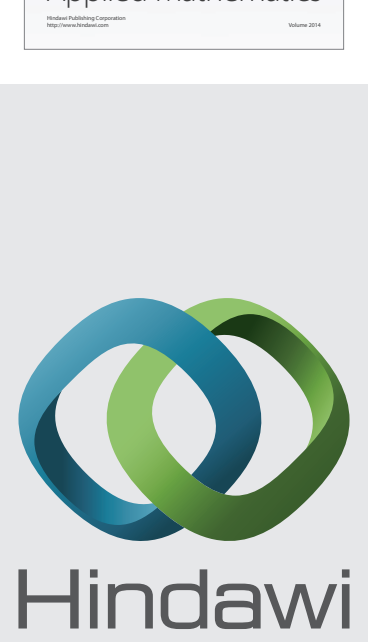

Submit your manuscripts at http://www.hindawi.com
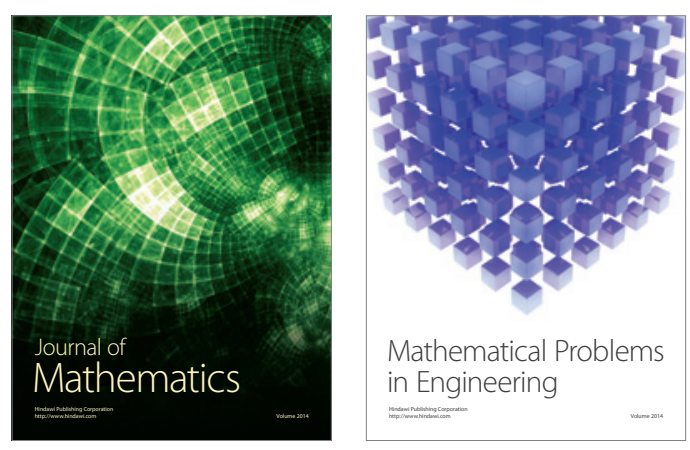

Mathematical Problems in Engineering
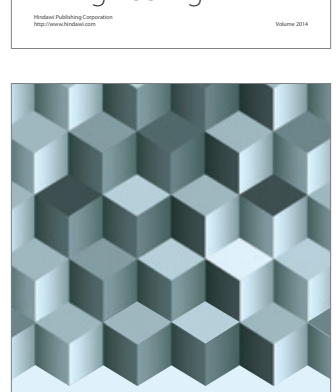

Journal of

Function Spaces
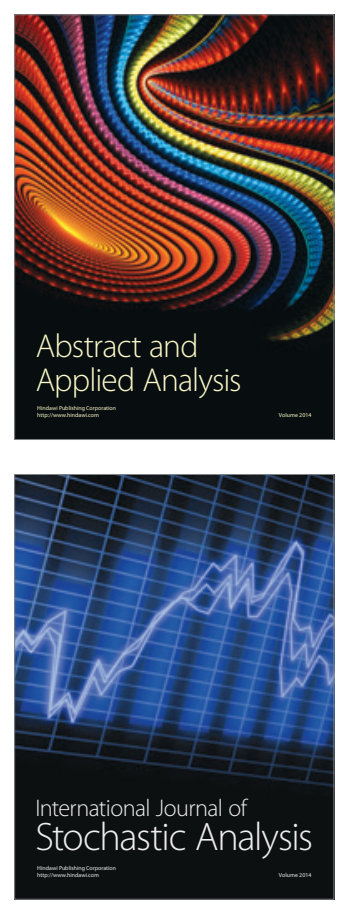

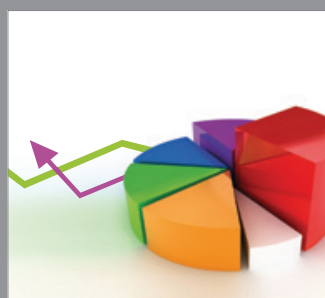

ournal of

Probability and Statistics

Promensencen
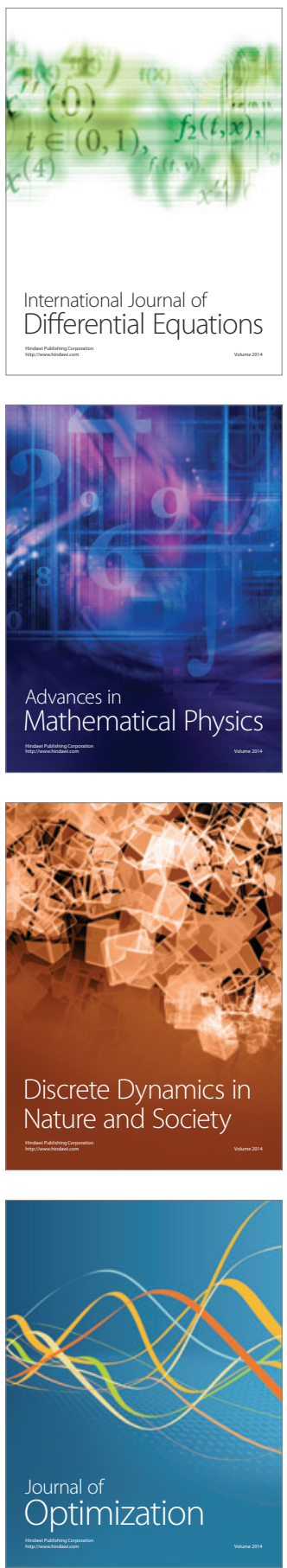